\begin{tabular}{ccc}
\hline & International Journal of Engineering \& Technology, $7(2.7)(2018) 326-330$ \\
SPC & International Journal of Engineering \& Technology \\
Website: $w w w . s c i e n c e p u b c o . c o m / i n d e x . p h p / I J E T$ & Research Paper \\
\hline
\end{tabular}

\title{
A new enhancement to avoid video distortion in wireless multihop networks
}

\author{
Dr. R. Bulli Babu ${ }^{1}$ *, SK. Shahid Afridi ${ }^{1}$, S. Satya Vasavi ${ }^{1}$ \\ ${ }^{1}$ Dept. of Electronics and Computer Science Engineering, KLEF, Vaddeswaram \\ *Corresponding author E-mail: shaikafridi567@gmail.com
}

\begin{abstract}
The protocols of typical routing algorithms which are designed for the wireless networks long back are specifically application oriented. Taking consideration at present, with the increase in the usage of the wireless networks the problem we are facing is video traffic. The very important thing is to maintain a good quality of video. At present users are opting videos in high quality that are to be delivered smoothly into their devices. The nature of video is changed due to 1) Because of the compression distortion occurs at the source. 2) With both interference and the errors induced on wireless channels distortion occurs. Hence, in this paper we work for the reduction of the distortion that occurs in video traffic. In order to overcome this problem we opted a wireless network in which the flow of application contains video traffic. For the clients the reduction of the distortion is very difficult. We cannot minimize the video distortions by using the link quality based routing measures. To understand this we construct an analytical framework first, then on the video distortions for accessing the impact. By using an analytical framework we design a routing measure for the reduction of distortion. With our experiment results we evaluate that our protocol is best for the reduction of video distortion and also for the reduction of the experience of user de-gradations.
\end{abstract}

Keywords: Analytical Framework; Minimization of Distortion; Routing Protocol Design; Video Distortion.

\section{Introduction}

Broadband and remote communication frameworks in today's world are more strong and omnipresent than they used to be prior. With the invention of the Smartphone video traffic became very famous. The maintainer of good quality of video is very difficult with both interference and the errors induced on wireless channels distortion occurs. In day to day life we watch wireless communi-cations happening in cellular and remote local area systems. This communication is watched as it were in the last two gadgets i.e. a base station and a remote end system. Multihop remote systems have one or many intermediate hubs which autonomously com-municate among themselves along the course and send or receive packets utilizing remote joins. Multihop systems can perform directing in a self-made way, since they don't rely on any past system base.

By this distinctive levels of outlines either i) the information encoded autonomously in case of I- outlines, or ii) encoding data is related to as of now encoded information in other outlines, as in case of P- and B- frames.

Primary basic -usefulness which is regularly dismissed over here is directing which impacts the overall nature of the video stream. In typical directing conventions, the streams are considered independently, they can merge onto certain joins that at that point ended up heavily loaded while others are underutilized. The choice of the flow made by such ordinary steering convention depends upon the network parameters.

Here we are primarily considered with the enhancement of the user perceived video quality by book keeping the application re-quirements. Here the plans which are utilized to put into code the clips of video can provide some amount of bundle misfortunes per outline by considering a limit for parcel misfortune per outline in the Group of Pictures (GOP). The misfortunes in the GOP are in stark -hop transmission. There is another credible sum of work differentiate with the conventional directing metric like an-ticipated communication result (ETX) [3] where the joins are eva-luated freely.

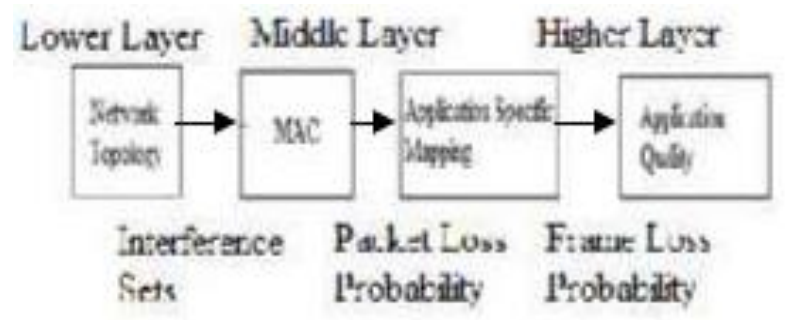

Fig. 1: System Architecture of an Analytical Model with Multiple Layer Approach.

Here the fall of the packet prospect on an associate is mapped to the prospect of a layout misfortune in the Group of Pictures which specifically associated with the video mutilation metric. As speci-fied over main problem in the remote sensor systems is directing, to resolve this issue we are taking after a energetic designing path that successfully holds the advancement of the process of frame-loss by utilizing which we are creating commonsense directing convention to minimize steering mutilation.

\section{Contribution of paper}

The development of an analytical framework is done in this approach that captures the affect of video mutilation and encourages the estimation of courses which will be ideal in rate of accomplishing the limited mutilation when video is transmitted end-to-end flow. We create a commonsense directing convention for a arrange 
which primarily carries the remote video by permitting the source to collect the twisting data on the joins and disseminating the traffic over the diverse ways in agreement to (i) the distortion, (ii) the outline position in the GOP.

By illustrating the viable steering convention through various extensive recreations and testbed tests we demonstrate that it is amazingly compelling in decreasing the video distortion end-to- end and making the client involvement corruption least.

\section{Related work}

Encoding and transmission of a video is dealt with in many ways and there are bounty of suggestions from different standardization bodies which oversee the encoding and transmission of video.

Unique video clip can be fragmented into a number of sub streams and transmitted over disjoint ways on a organize. This strategy to fragment an unique video clip and at that point transmit is called Multiple Portrayal Coding (MDC). Interpreting prepare of the unique video clip can be fruitful utilizing the descriptions sent on the arrange also video quality of is moved forward in large amounts with decoded sub streams. Layered Coding is another strategy to send and improve the video quality. Different upgrade layers along with a base layer are utilized in this method. Layered Coding is embraced in this paper due to its notoriety in applications and benchmarks embraced [4], [5], [6].

Different levels of encoding are to isolated unique video clip into a cluster of outlines of distinctive needs with respect to quality.

These are called I-, P- and B-frames. A structure named GOP (Gather of Pictures) comprises of group of such outlines. I-frame is the starting outline in each GOP which is decoded freely from the same GOP without any other data. To translate other outlines Pframes can too be used as a source [4].

The flag preparing experimentation community is the pioneer in conducting inquires about on outline - misfortune - resilient video [9]. Unique video is partitioned into tall and low priority outlines and tall need outlines are secured by FEC in [10]. Due to quantization and outlines misfortunes there is temporal and spatial mistake presented in the video stream. To calculate the mutilation presented in the video an algorithm is proposed in [11] which is utilized for switching in the middle of connects also each macroblock for intraoding. This results in larger Crest Flag to Clamor Proportion (PSNR) [4]. Rate distortion optimization plot is utilized to decide the coding parameters. Reenactments are conducted to evaluate these plans. Reenactments are done with a reliable frame-loss rate to watch the impact on network transmission. The characteristics of genuine world frameworks are not captured in such reenactments [12].

To think about the impacts of remote channel blurring on video distortion a system is outlined in [13] which it is only credible for single-hop transmission. There is another credible sum of work performed on a single interface in [14]. Experimentation is done to consider the impacts of outline loss and how much of twisting can be dealt with by the compressed video with regard to the length of mistake in a frame. A two dimensional Markov chain framework is introduced after looking at the accomplishment of video stream-ing over multihop 802.11 remote arrange. Conclusion to end QoS is arranged and conveyed in video spilling model of two dimen-sional Markov chain along with performance evaluation. To un-cover the normal twisting transmitted along sequential P-frames is defined utilizing a recursion model in [16]. Affect of directing on video mutilation is not considered or done in any of the over in-quires about. Remote 4G systems are too utilized to look at the performance of video transmissions since they have support high Quality of Benefit for video transmission. H.264/SVC encoding is inspected over portable WiMAX [17]. Quality of Service which is experienced by the conclusion client is represented by measurements such as PSNR and MOS. Conclusion is that the performance is subordinate on different encoding schemes used in conventions and responds in an unexpected way to the misfortune of frames in arrange. Once more the affect of directing on video distortion is not considered or done in any of the over investigates.

Steering calculations for Quality of Benefit and cross layer optimization on remote advertisement hoc and work systems is researched broadly [1], [2], [3], [18]. Based upon protocol assessment measurements such as transport/application, network and MAC layer measurements, QoS can be separated in several ways.

A overview of the same is done in [19]. Performance measurements particularly characterized for video transmission is not taken into account in any of the routing schemes displayed in the studies. The applications require to indicate throughput and delay limitations indeed when a routing plot with QoS is characterized.

In our approach, video distortion metric which is related to application performance metric is specifically coordinates into the route selection framework.

To move forward the Quality of Benefit multipath routing schemes are utilized in video transmission and directing is focussed on Different Portrayal Coding [20], [21]. Disjoint ways are calculated utilizing data collected at the goal hub and this is an expansion of Dynamic Source Steering which is utilized to back multipath video transmission. The steering plot outlined here is based totally on reenactment without any investigation [20]. Disjoint paths in are calculated by planning a given set of path lengths and there is no execution metric defined directly with video quality and instep delay imperatives are used in the optimization. Minimization of in general video distortion is accomplished by selecting steering ways properly. This is characterized utilizing a rate twisting demonstrate and utilized in an optimization issue.

Remote advertisement hoc systems utilize MDC for video multicasting. Determination of courses using optimization issue is a complex issue, consequently a heuristic based calculation is utilized to calculate the courses. The models utilize MDC to take into account the twisting of the video and we utilize LC approach along with contrasting on the way we demonstrate video distortion.

802.11 remote work systems utilize multipath routing scheme for conveying video stream and it depends maximum on disjoint ways to pick up great activity designing.

In a progressive demonstrate is utilized to plan a routing scheme for vitality proficient video transmission with minimum QoS corruption for LC. Such hierarchical models depend on hubs which are combined into the clusters also into an intermittent handle of choosing a cluster head takes place. This increments authoritative outlines on the network thereby expanding the preparing and information communication. A demonstrate where all hubs of a organize perform the same set of assignments and are rise to without any pecking order is proposed in our plot.

\section{System analysis}

\subsection{Existing system}

There are different kinds of approaches that exist in dealing with transmission and encoding. There is a MDC procedure which parts an introductory clips of video into various large amounts of small parts which are called as descriptions.

MPEG-4 and H.264/AVC give rules to encode the video clip for transmission over a framework of communication that is related on the basis of codlings of layer called layered coding. Regularly, starting clip of the video will be isolated in grouping outlines of diverse significance that regards quality, subsequently encoding of diverse levels.

According to another existing demonstration, the explanatory system which created for showing impacts of the channel remote blurring on the distortion of videos.

According to another existing demonstration, creators look at impacts of the loss of packet designs also particularly height of the mistake forms in the mutilation of the video compressed. 


\subsection{Disadvantages of existing system}

According to the client's point of view, keeping high quality exchanged video which becomes basic.

Quality of the video is influenced with: 1) mutilation that occurs with the compression at the source, also 2) twisting occurs with the interference and the remote channel initiated blunders.

The demonstration is substantial or the single ho communication.

\subsection{Proposed system}

The proposed system is the client's awareness on the video quali-ty is essentially progressed with the bookkeeping necessities of applications, also particularly twisting of the video is experienced with a stream. Regularly, plans utilized for decoding the clips of video is obliging the amount of parcel misfortunes for each outline. Be that as it may, in the event that the number of misplaced par-cels in a outline surpasses with a limit, outlining is not possible to decode accurately.

The outline misfortune results into a few number of the mutilations. An esteem in which the twisting which is made at the bounce beyond the way which is depending on the unrecoverable positions of the outlines of the video(basically alluded with out-lines).

According to the fundamental commitments, the development of an expository show which is characterizing the energetic character to prepare portrays for the advancement to outline misfortunes (instep with fair centering with the arrangement of the metric of the quality like fall of the packet likelihood) because the convey of the video is on end-to-end way.

In this system, we want to show how the way to a every end-to-end stream influences execution of that streams with respect in the terms of the video distortion. It is built on the basis of the mul-tilayer approach.

\subsection{Advantages of proposed system}

Arrangement with issue which is taken into the basis on energetic program the way to achieve this is that viably takes advancement of the loss of frame process. Minimizing the distortion directing.

The loss of many I-frames which carry out the most essential data will effect the distortion the most, Since our design says that the frames that to be send to the paths which experiences the least amount of congestion.

The directing conspire which is optimized for exchanging clips of video into the remote systems with the least distortion.

\section{Implementation}

The implementation is done through different modules for the resistance of video distortion framework is.

\subsection{Model formulation}

Here the explanatory demonstrate couples the physical and Media Access Control sheets to organize with one of the layer named as application layer for the clips of video which are sent from the input to the goal hub. This shows that the lower layers results the packetfall likelihood along a multiple collection of conditions which demonstrate the multiple client impedances, conditions of the physical paths, also the activity shows in middle of source node-destination node. Packet-fall likelihood which acts as an input to the model second for computing the packet-fall likelihood, and also the desired mutilation. Twisting esteem depends upon the to begin with unrecoverable outline in the GOP along the path from source to goal at a specific bounce.

\subsection{Video distortion model}

With the detailed examination the video distortion communication is a breakdown into source distortion in a model and also the dis- tortion of wireless transmission with a single hop. we develop a model to capture the assessment for transmission mutilation along the joins of a course from source hub to goal hub. In this model we determine a GOP pattern which comprises of I- outlines taken after by $\mathrm{P}$ - frames then we compares the I- outline list with, and the $\mathrm{P}$ frames corresponds to list to 1 up to $(\mathrm{F}-1)$. The distinctive outlines are expecting the packet losses in the GOP are free occa-sions, can be computed the transition probabilities for the prepare.

\subsection{Video distortion dynamics}

Nodes from source to destination at hop the distortion of the value depends on the unrecoverable frame position first in the GOP. The loss of the first I-frame is shown by the value 0 , and then GOP cannot be recovered. The value in the range o (F-1) and 1 represents that particular P-frame which is the starting frame in GOP which is un decoded perfectly, those values represent that no frame was lost as far.

\subsection{Optimal routing policy}

The main goal is to discover the way which provides least carry-ing mutilation in the middle of source and destination. The control to the optima control issue is the determination of the next hub to be gone to at each middle node from the source to the goal. In quintessence, the MDR routing policy disseminates the video outlines and the parcels along the multiple ways by minimizing the impedances experienced by the outlines that are the starting of the GOP. Than other outlines I-frames are the longer outlines, so the misfortune of those outlines results in overwhelming twisting, and hence the transmission is on relatively interference-free ways.

\section{Execution results}

For achieving the less video distortion the protocol contains these forms.

Form of Router.

Form of Node.

Form of Server.

Case 1: When the request for the video file has been sent from the Source node(Normal node to the server.

1 Model formulation

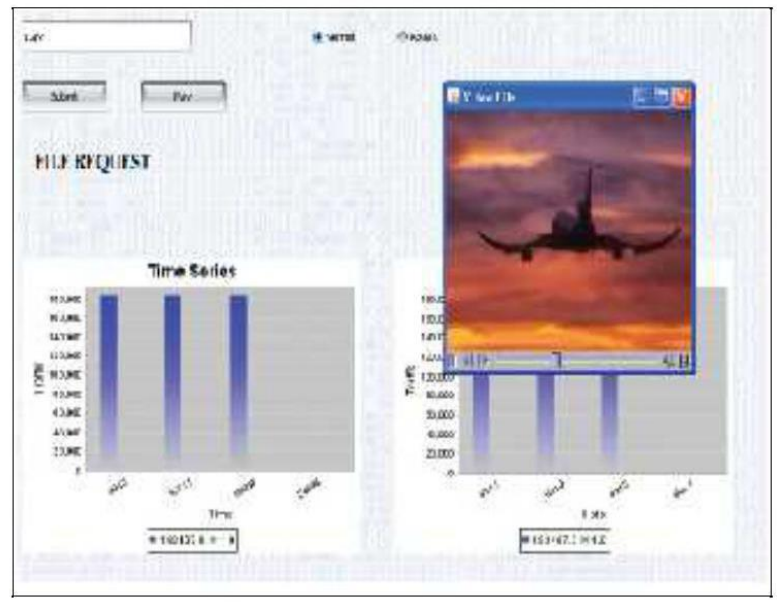




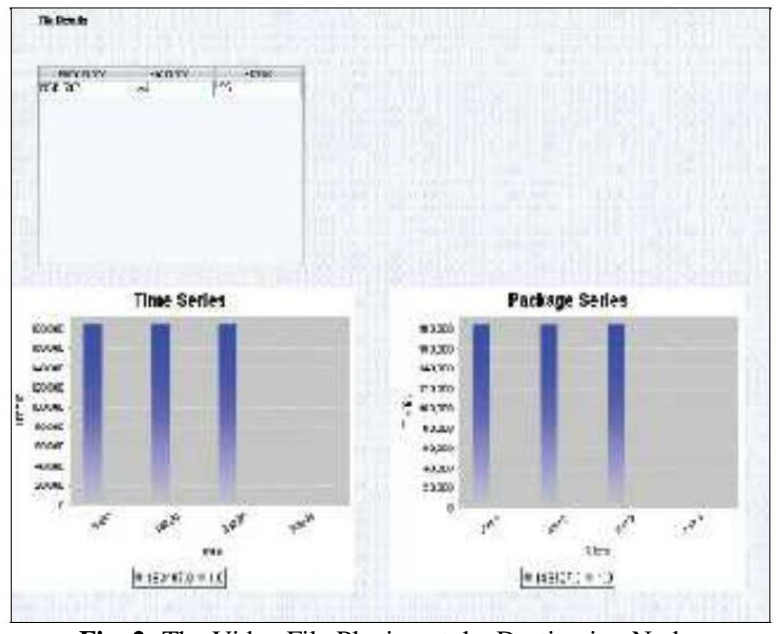

Fig. 2: The Video File Playing at the Destination Node.

Case 2: When the request for the video file has been sent from the Source node(Attacker node) to the server.

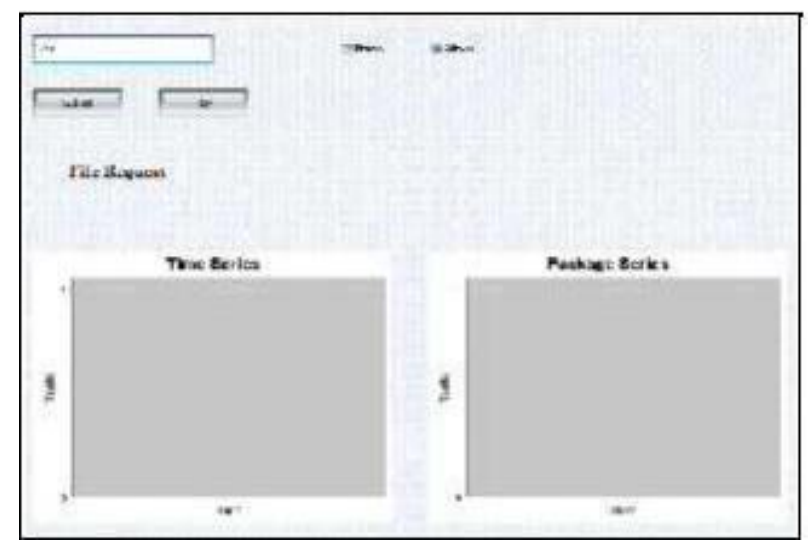

Fig. 3: Selection of Attacer Node and the Video File in the File Reques Form.

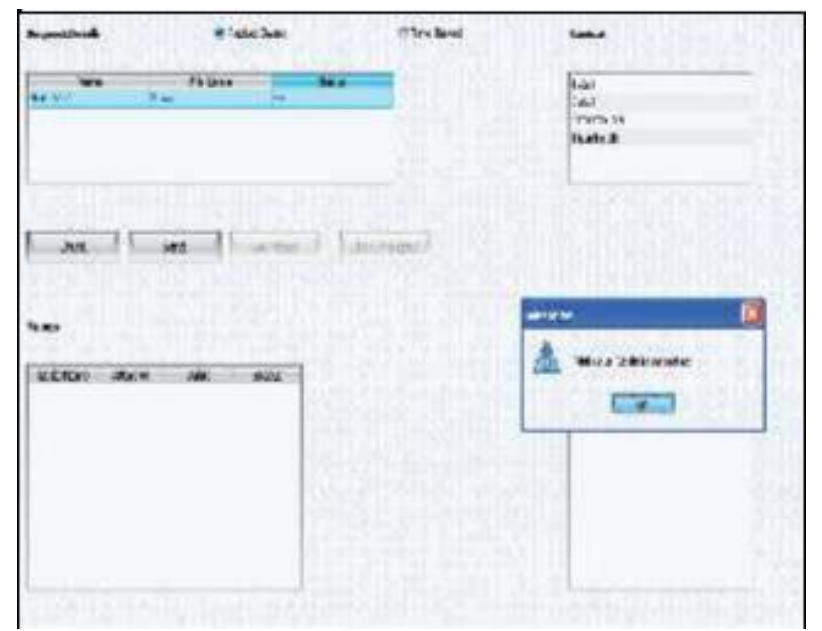

Fig. 5: Time Series and Package Series in the Router Form.

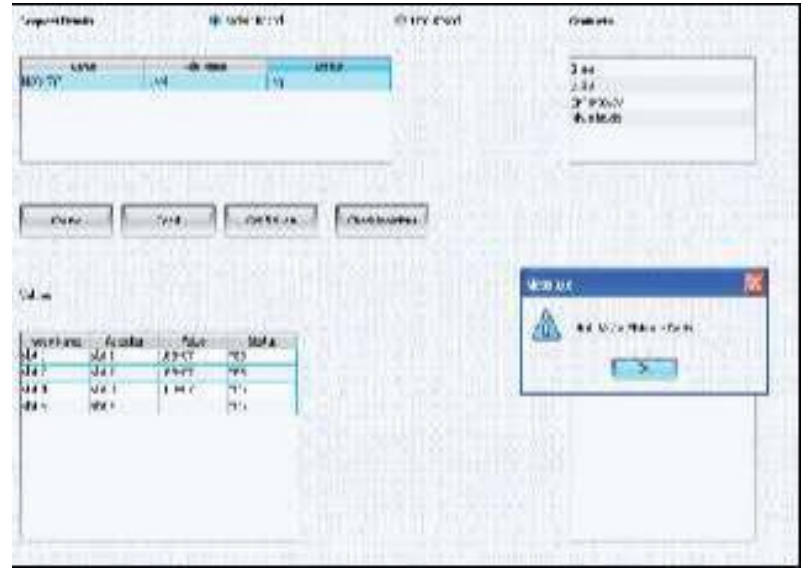

Fig. 6: NOD 535 has been Detected Has Malicious Node.

\section{Conclusion}

Due to the imperfections and downsides of existing framework in providing mutilation free video an Explanatory framework was created utilizing multihop remote organize to reduce the mutila-tion while sending the video from source to destination. The Ex-pository system employments the newly designed twisting resis-tance directing convention for minimization of mutilation in the video. The Proposed model moves forward the video quality by minimization of the distortion.

\section{Future scope}

Numerous recordings can be sent to goal hub at a time. The Content message sent along with video can be encrypted using figure content strategies.

\section{References}

[1] Sadia Suroor,Manoj Challa, Zafar Ahmed Syed, "Enhance-ment to Distortion-Resistance Routing ramework for Video Traf-fic in Wireless Multihop Networks, International Journal of Com-puter Science and Information Technologies, Vol.7(3), 2016.

[2] Koucheryavy,Y.,Giambene,G.,Staehle,D.,BarceloArroyo,F.,Bra un,T.,Siris,V," Quality of Service management in the wireless multihop networking and Traffic", Vol 31, 2016

[3] Viraj S.Ambedkar, James W.Modestino "Enabling Real-Time H.264 Video Services over Wireless Ad Hoc Networks Using Joint Admission and Transmission Power Control", Vol 28, Feb 2005.

[4] Shailendra, V. Srikanth Krishnamurthy," The Distorting Routing Resistance framework to reduce the video traffic in the wireless multihop networks", on Networking, volume 23, Apr 2015

[5] K. Susmitha, T.V. Naga Jayudu"A Routing policy or mini-mizing distortion for video traffic in wireless multihop networks, Vol4, 2016.

[6] J.Chakareski S.Han and B.Girod" Multiple Descriptions vs Layered Coding over multiple paths for video streaming," volume 10, Apr 2005.

[7] J.Jayashree, 2R.Dhandapani, "throughput analysis of a path in routing framework for video traffic in wireless network", Interna-tional research journal in advanced engineering, vol2 issue 3, june21, 2016.

[8] Wiegand.T, Sullivan G.J, Bjontegarrd, "Systems and Circuits for the video Technology on IEEE Transactions," volume 13, 2003 July.

[9] Suresh Kumar T, B Madhusudhana Rao,'Transmission in the mobile ADHoc Networks and Click Prediction for video stream-ing", vol6, 2017.

[10] George Papageogiou, Shailendra Singh,” The Distortion Resistant Routing Framework to avoid video traffic in Multihop Networks", Volume 23, Apr 2015.

[11] Zhang Rui,L.Shankar Regunathan and Rose Kenneth," Video Coding with Optical Inter/Intra-Mode Switching for Packet Los-sResilience," ieee journal on selected areas in communications, vol 18, june 2000.

[12] Jimin Xiao, Tammam tilloemail author, Chunyu Lin and Yao Zhao," The Resilient video coding with the end-to-end Rate Dis-tortion Optimized at the macrolock level, Advances in Signal Processing in the journal of eurasip, 2011. 
[13] Shaik Mohammad Arif Ali and Sayeed Yasin," A New Ap-proach for the Framework Video Traffic", IJETCSE International Journal of Engineering and Technology, volume 23, Nov 2016.

[14] Liang Yi J, G. John Apostolopoulos," Packet Loss for the compressed video analysis",ieee transactions, volume 18,July 2008.

[15] A.K.G. Dhana Lakshmi, T Sivarama Krishna," a routing framework for video traffic, IJET, Volume 5, 2017.

[16] Wang Yao, Wu Zhenyu and M.Jill Boyce,'Transmission loss Induced Distortion modeling in Decoded Video, IEEE Transac-tions, volume 16, june 2006.

[17] Migliorini Daniele and Mingozzi Enzo," The evaluation of the performance video streaming", International Jouranl of com-puter and Teecommunication, Volume 55, 2011.

[18] Shubat S.Ahmeda and Reda K. Farah," Performance study on the routing protocols for mesh wireless networks", Congress of International and Engineering on communication and computers, volume $59,2014$.

[19]Hanzo and R.Tafazolli,"'Routing Solutions survey for mobile and ad hoc networks, Surveys on ieee communications, volume 9, Apr 2007.

[20] J Chen and SHG Chan," Multipath routing for the video delivery over networks", ieee journal, volume 22, 2014. 